\title{
IOT BASED WIRELESS ATTENDANCE MANAGEMENT SYSTEM USING FINGER PRINT RECOGNITION
}

\author{
D.Narendharsingh1, Anusha Reddy2 and Dr.Sharma Sudhir Kumar
}

Abstract Attendance is for keeping records of number of students present in schools, colleges or in any organization. It is very important strand in maintaining discipline among employees in an organization and imparting quality education in schools, colleges and if someone drifts from required standards proper action can be taken. Complexity of this process increases even more with increase in number of students. In proposed system two hardware devices are there, first one is a handheld device which will be there in every classroom for taking attendance and second one will be a local server for all handheld devices in the network. Handheld devices are designed on Arduino microcontroller, 16x2 Liquid Crystal Display (LCD), Zig-Bee module, Fingerprint module. Local server is Raspberry Pi web server interfaced with Zig-Bee series 2 module based on an IEEE 802.15 standard.

\section{Introduction:}

This paper is organized as follows: Section I consists of Arduino and Section II deals with Raspberry Pi.Initially fingerprint of students should be stored in the server to recognize the student. For that we have to use enroll (E) button for enrolment. By pressing $E$ the fingerprint of a particular student is stored in the data. Like-wise we have to store all the fingerprints of the students.

During the process of taking attendance we need to press start button (ST) to start the process. A green LED is glowed to know the process is started or not. After the process starts, the student should press Identification (I) button before he keeps his/her finger for attendance. The process should continue till all the students completes their identification. If all the students completed their attendance the teacher should stop the process the pressing Stop (SP) button and it indicates with the red LED in the board. All the above process is done in raspberry Pi board except the fingerprint module. The fingerprint module is connected to Arduino and the serial communication is done through $\mathrm{X}$-bee modules which is connected to both raspberry and Arduino.

After the process ends the data/attendance for that particular day is being uploaded to server and a message is to be sent to the students through a SMS by using GSM module which is connected to the raspberry pi. In the server the data is stored in a file

\section{BLOCK DIAGRAM:}

\section{RPI SCTION BLOCK DIAGRAM:}

Finger Print Section:

\footnotetext{
${ }^{1}$ AGI, Hyderabad

${ }^{2}$ Anurag Group of Inst.

${ }^{3}$ Jaipur National University, Jaipur
} 


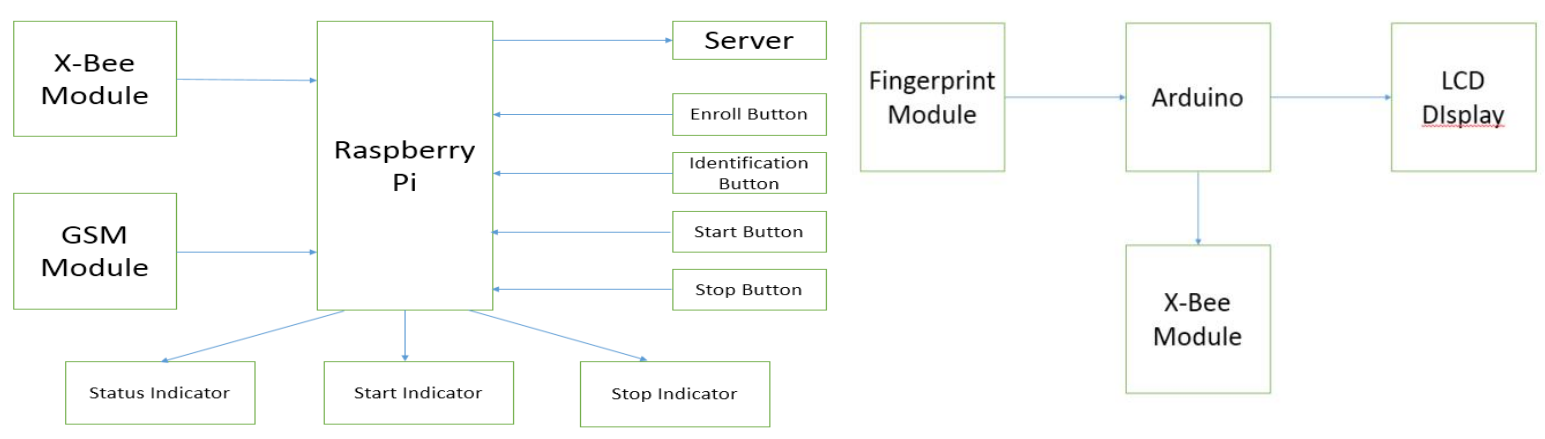

Initially fingerprint of students should be stored in the server to recognize the student. For that we have to use enroll (E) button for enrollment. By pressing $\mathrm{E}$ the fingerprint of a particular student is stored in the data. Like-wise we have to store all the fingerprints of the students.

During the process of taking attendance we need to press start button (ST) to start the process. A green LED is glowed to know the process is started or not. After the process starts, the student should press Identification (I) button before he keeps his/her finger for attendance. The process should continue till all the students completes their identification. If all the students completed their attendance the teacher should stop the process the pressing Stop (SP) button and it indicates with the red LED in the board. All the above process is done in raspberry Pi board except the fingerprint module. The fingerprint module is connected to Arduino and the serial communication is done through X-bee modules which is connected to both raspberry and Arduino.

After the process ends the data/attendance for that particular day is being uploaded to server and a message is to be sent to the students through a SMS by using GSM module which is connected to the raspberry pi. In the server the data is stored in a file.

\section{FLOW CHART}

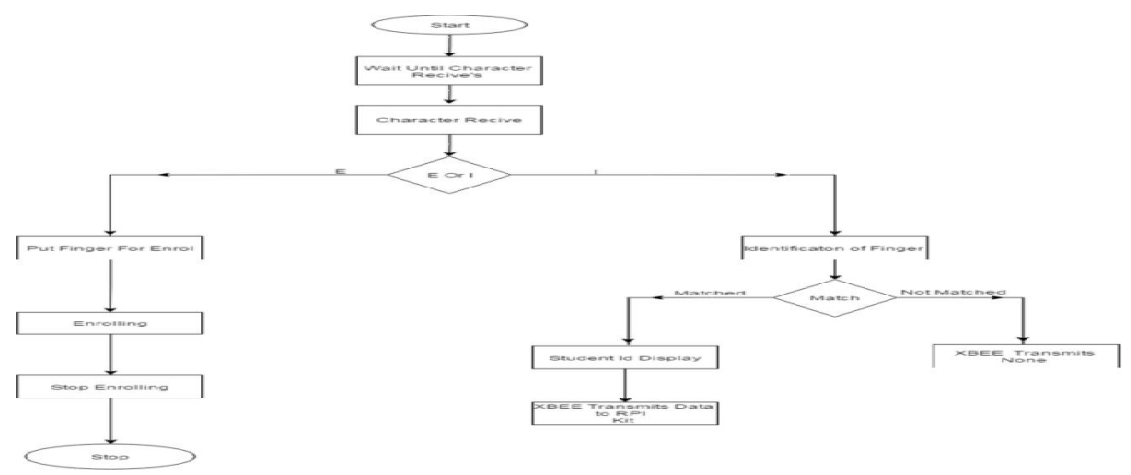

\section{Fingerprint Recognition:}

In the context of the term "identification through fingerprint images", fingerprints are generally accepted as human fingerprint images. Identification can functionally be split up into the following three basic tasks:

- Fingerprint scanning,

- Fingerprint classification and 
- Fingerprint comparison.

Fingerprints can be acquired as color prints or via sensors which store the ridges on a medium (glass, paper, sensor surface, etc.). During the classification process, fingerprint images are optionally allocated to a certain category based on the global orientation of the ridges while the location of the minutiae is marked as well. The comparison determines whether two fingerprint images are identical, i.e. whether they belong to the same person (finger). The complete process of a fingerprint image analysis (comparison of patterns) can be dividedInto six steps as shown in the below figure.

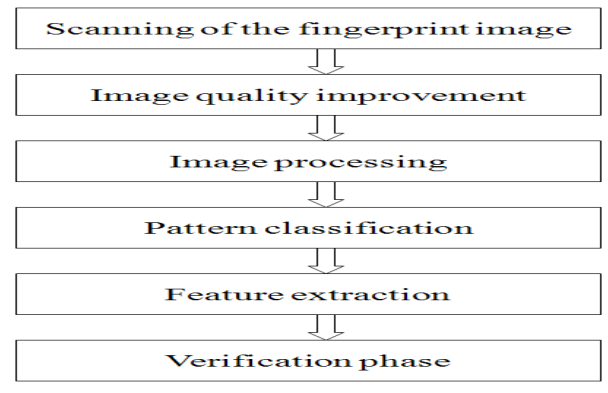

\section{Process of fingerprint analysis:}

1. Scanning of a fingerprint image. The quality of the scanned image is the decisive factor for automatic identification purposes. It is desirable to use a high-definition fingerprint scanner which is able to tolerate different skin types, damages, dryness, as well as the humidity of the finger surface.

2. Image quality improvement. By using image quality improvement, an optical improvement of the structures (ridges) on the scanned image can be achieved.

3.Image processing. Image processing means the preparatory phase for feature extraction and classification purposes.

4. Feature classification. The fact is that all fingerprints show certain global similarities, which allow for rough classification into three principal finger classes. However, classification is a rather difficult process both for algorithm-based decisions as well as for man-made decisions since some fingerprints cannot be clearly allocated to a concrete finger class. Nowadays, pattern classification is only used in dactyloscopic systems, e.g. AFIS (Automated Fingerprint Identification System) of the Federal Office of Criminal Investigation (BKA). This method is not feasible for access systems.

5. Feature extraction. In this phase, the location of the minutiae (ridge bifurcations and ridge endings) in the fingerprint is detected and extracted. In practice, scanned fingerprint images show differing qualities. The algorithm performance is negatively influenced by a poor image quality.

6. Verification phase. In the verification phase two feature vectors are being compared. The algorithm performance strongly depends on the quality (significance) of the extracted minutiae and on the comparison process.

Below, we will describe in more detail the fingerprint scanning, feature classification and fingerprint comparison processes.

\section{Fingerprint Scanning:}


Depending on whether the scanning process is carried out on- or off-line, the fingerprint image can either be

- A color image, e.g. on paper or

- An image of a life finger obtained through a sensor.

In case of a color print, rolling the finger on a surface generates the image of the ridges, e.g. on paper after that the finger is moistened with ink. An example of such rolled fingerprint images can be seen in the figure. By evenly rolling the finger from one side of the nail to the other, all line-related data is reliably recorded in the image. Afterwards, these images can be scanned or electronically photographed. In dactyloscopy, this method has already been used for well over 100 years. Thus, since a complete "overall imaging" of the finger is done, in addition to a higher number of ridges and minutiae, "macro features" (i.e. delta and nucleus) are recorded as well. Even though they are part of each and every ridge pattern (with the exception of the "arch" pattern which does not have a delta), they are not always printed.

A disadvantage of this method is a possible distortion, which may occur through pressing and rolling the finger while taking the fingerprint. Furthermore, a quality feedback is not possible which may lead to a decrease in the quality of the fingerprints. From the user's point of view, this is an unpleasant and slow method. It is also unsuitable for partially automated access controls.

The term life image of a finger is a comprehensive term for images that are obtained directly by placing the finger on a suitable sensor. There are a vast number of various methods, which can be used for

scanning ridges. They include:

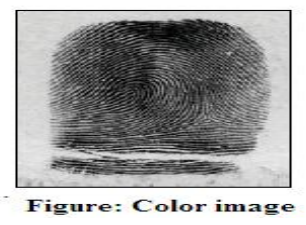

- Optical sensors.

- Electrical field sensors,

- Polymer TFT sensors • Thermal sensors.

- Capacitive sensors.

- Contactless 3D-sensors and

- Ultrasound sensors.

A biometric sensor is the hardware component of a biometric system, which initially supplies biometric measurements. Depending on the biometric method in use, there are different kinds of sensors. Optical sensors use light for obtaining fingerprint images. Electrical field sensors measure local variations of the electrical field, which is generated on the finger surface relief upon the emission of a small electrical signal. Polymer TFT sensors measure the light, which is emitted upon contact when the finger is laid on the polymer substrate. Thermal sensors register the thermal finger image. In capacitive sensors, the sensor and the finger surfaces together form a capacitor. The capacity thereof changes based on the skin relief (skin ridges and grooves). These local changes are measured and thus represent the fingerprint. 
The above-mentioned sensors are used in connection with the data-processing module as on-line systems. They substitute the off-line method in which fingerprints are, for example, taken on paper before they are digitized later on. Image quality strongly depends on the "contrasts" that were achieved between the ridges and the adjacent grooves. Since there is a feedback to image-processing algorithms for on-line methods, it is relatively easy to immediately check the quality of fingerprint images that were just obtained.

The life image is usually recorded by lightly placing the finger on the surface of the sensor. Since it is not so user-friendly, the finger's surface is only rolled in the context of AFIS-systems (as in the dactyloscopic method). Of course, in this case, only such ridges can be captured that are recorded as a result of being directly in contact with the sensor surface. Hence, compared to rolled fingerprint images, the life image generates the image of a smaller section of the finger's surface but, in addition, it might also have smaller distortions of the image.

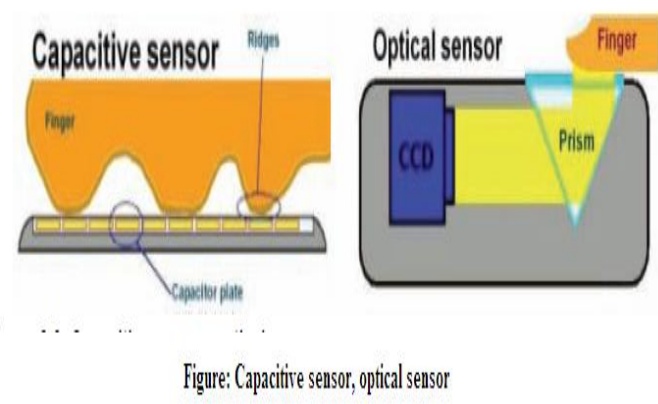

Currently, the most frequently used life image technology is the optical method. Upon placing the finger on the sensor's glass pane (prism), the elevations of the papillary lines are in contact with the glass; the grooves, on the other hand, are not in contact with it. Basically, the recording device consists of a light source (LED) and a CCD camera, both of which are located within the device on the other side of the glass pane. The light of the LED illuminates the glass at a certain angle and the photo-element receives the reflected light. The course of the beam runs in such a way that the incoming light on the contact ridges is scattered as if on a mirror surface and then reflected back on the CCD camera. There where the grooves are behind the glass pane, the light passes through; these spots remain dark. An example for such a fingerprint image can be seen in the figure shown below.

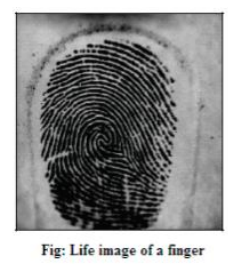

\section{HARDWARE IMPLEMENTATION}

\section{Design Aspects}

We are connecting Arduino Ports A0, A1, A2, A3, A4 with LCD.

ZigBee -1 Module TxD and RxD are connected to Arduino TxD and RxD Wise versa.

Fingerprint Module haveTxD and RxD those are connected to Pin-2 and Pin -3.

The Raspberry PI Module's TxD and RxD are connected to GSM TxD, RxD wise versa. 
The ZigBee-2 Module is connected to Raspberry PI through serial connecter.

The GPIO Header of RPI Module is connected to LED SECTION and switch board.

\section{Design Approach:}

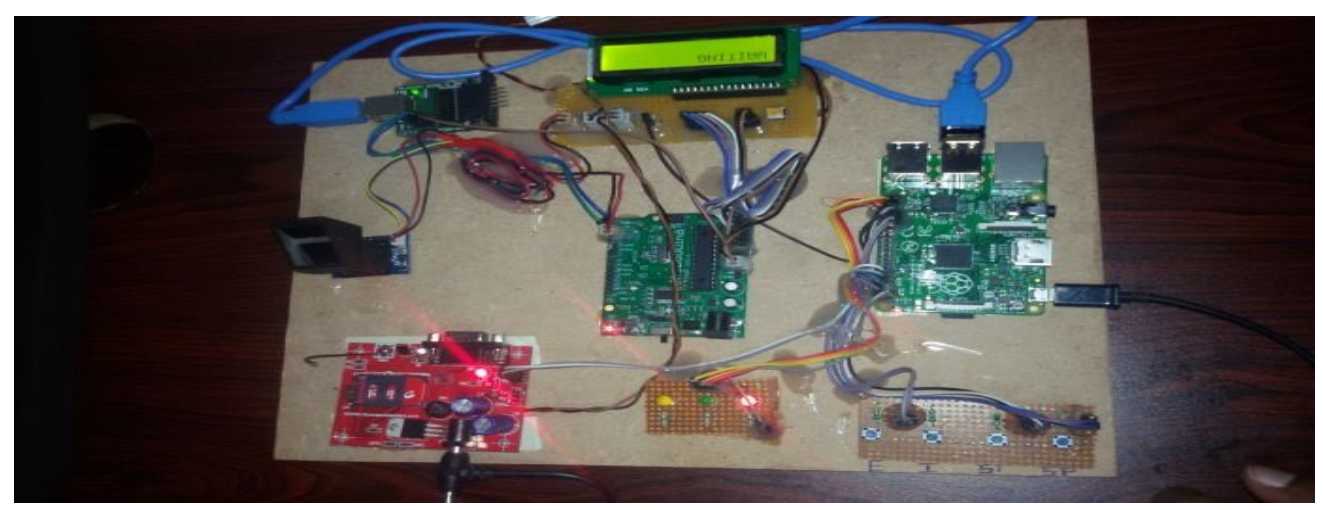

\section{GSM Module:}

1) Insert SIM card open the SIM cardholder by sliding it as per the arrow mark and lift up. Insert the SIM card, so as to align the chamfered corner suits in card holder .After inserting the SIM card, lock the holder by sliding it to the opposite direction of arrow mark.

2) Connect the antenna fix the supplied RF antenna to the SMA Antennae connector and tighten it by rotating the nut (Never rotate the antennae for tightening).

3) Connect the pins connect the GSM modem as per the circuit diagram provided

4) Power the modem power the modem from suitable power supply, which is having enough current capacity (>1A).

5) Check the Status of the LEDs PWR LED - Red LED will lit immediately STS LED - Green LED will lit after 1-2 seconds NET LED -Blue LED will starts to blink in fast for few seconds(Searching For Network) and becomes slow blinking once the Modem registers with the Network.

6) Network LED the network LED indicates the various status of GSM module eg. Power on, Network registration \& GPRS connectivity. When the modem is powered up, the status LED will blink every second. After the modem registers in the network (takes between 10-60 seconds), LED will blink in step of 3 seconds. At this stage you can start using modem for your application.

7) Baud rate The Baud rate supported by the modem is between 9600 and 115200. Make sure the host system is set to the supported baud rate. The modem automatically sets to the baud rate of the first command sent by the host system after it is powered up. User must first send " $A$ " to synchronize the baud rate. It is recommended to wait 2 to 3 seconds before sending "AT" character. After receiving the "OK" response, Your Device and GSM Modem are correctly synchronized. So there is no need for setting the baud rate using commands. Before You Start using the modem, please make sure that the SIM card you inserted support the needed features and there is enough balance in SIM.

\section{Test plan:}

i) Initially give power supply for RASPBERRY PI module and also other modules. 
ii) After that we have to wait until RPI Boot Loader processed and should be connected with Wi-Fi module.

iii)Press the Enroll button and wait, until ZigBee module Transmits Character 'e'.

iv) When the Arduino XBEE receives the ' $\mathrm{E}$ ' the person can able to Enroll with

v) Finger print through module, it can take image and stored it in RPI.

It Display's student enrolled successfully.

vi)It generates student ID. After completion of Enrolling.

vii)Stop Enrolling by pressing stop.

viii)Press the start button, To Identify.

ix)Press the 'I 'Button for Identification with Fingerprint Module.

$\mathbf{x}$ )If Identified, it gives student Id.

If not, it Display's none.

xi)After completion of Identification of all members, press stop for further process.

xii)GSM Module will Send SMS's for registered mobiles as per attendance.

xiii)AURDINO will send whole data thorough serial port to the RPI.

xiv)RPI module upload's the whole data through Wi-Fi module to the respected server in the text file.

TEST RESULTS

Press
identification

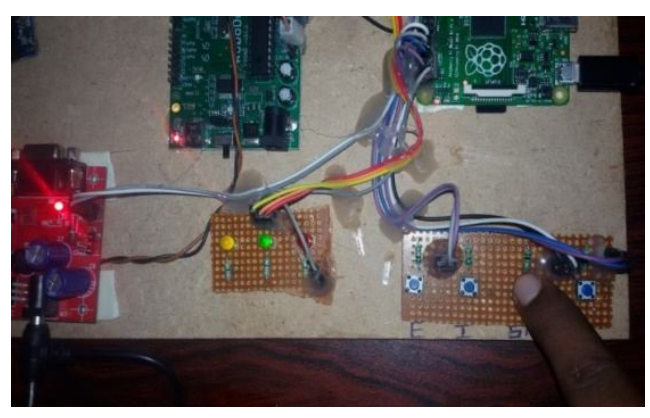

Fig 1
LCD displays "waiting"

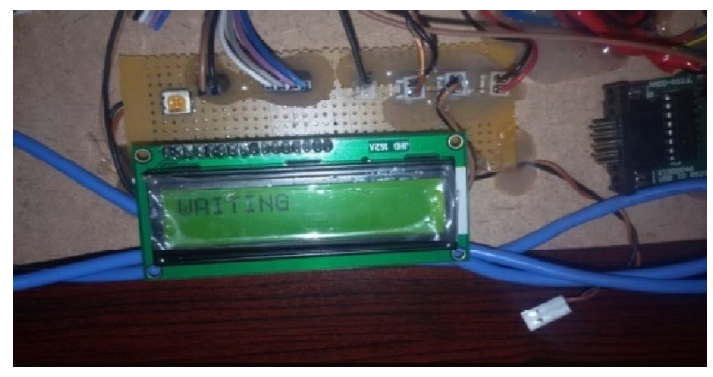

Fig 2

LCD displays after enrolling 


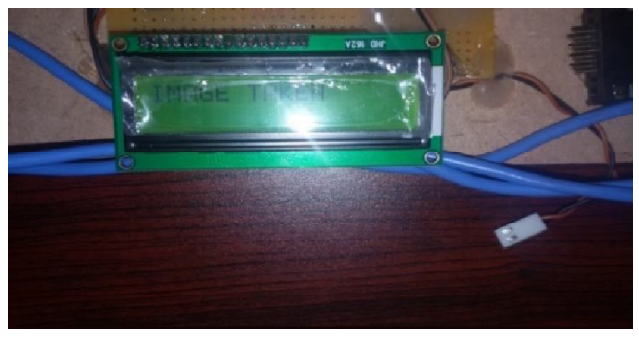

Fig 3

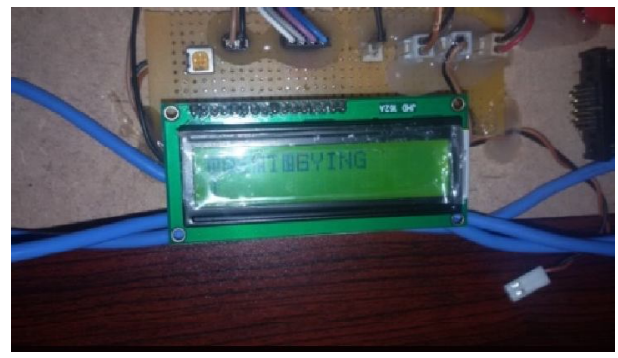

Fig 4

\section{Identification process initiated.}

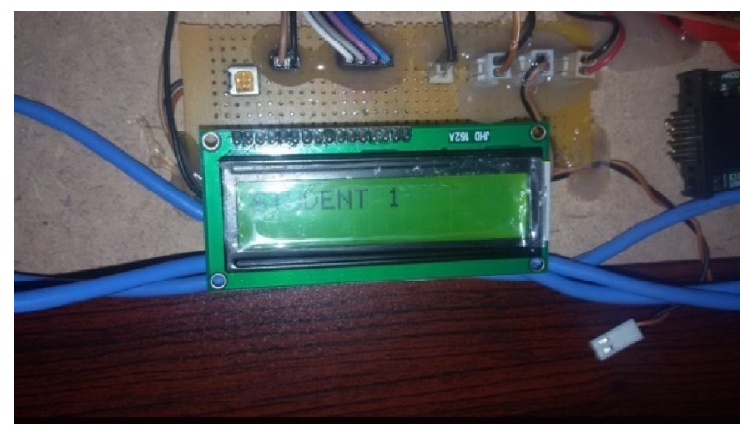

Fig 5
Identified student fingerprint displays ID of that person

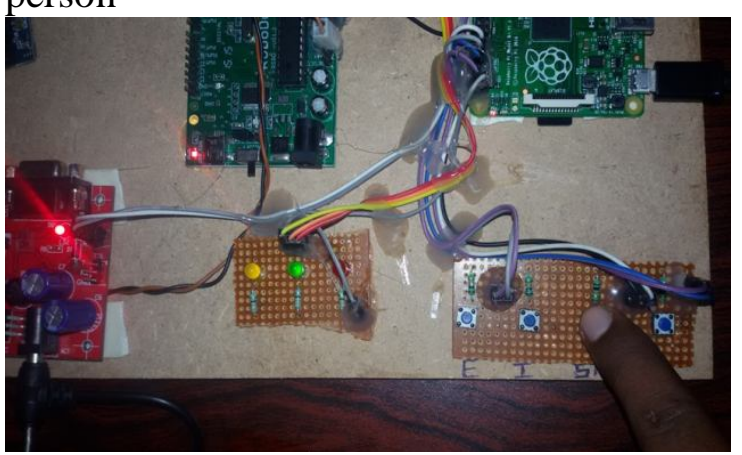

Fig 6

To press stop button terminating identification process

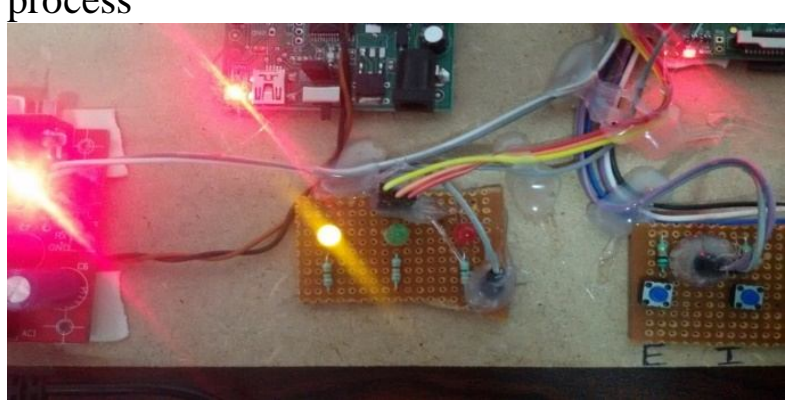

Fig 7

Taken attendance being uploaded to Cloud storage.

\section{ADVANTAGES:}

1) It reduces the complexity, when taking Attendance from class or in any company or Organization.

2) Finger prints are unique in nature, it gives attendance for only those who are enrolled and gave fingerprint while attendance time.

3) It avoids duplications, so cheating is Impossible.

4) No need of calculations and other manipulations at end of the month or year.

\section{CONCLUSION:}

To maintain the attendance system with Fingerprint Recognition. That means using biometric like fingerprint module. As fingerprints are unique in nature.

The main aim is to retrieve the complexity in attendance system, when no. of students increased in school or Library. We can avoid cheating and maintain discipline in all environments. 
Here we are using RASPBERRY PI with ARDUINO and GSM implementation ZIGBEE is used for transmission.

\section{FUTURE WORK:}

Our future is depending upon Embedded System. Here we can implement with other biometrics' application like palm scanner for door opening, Face scanning for attendance system, with the help of RPI and ARDINO with GSM. Here the hardware is low cost, Software is Linux, and platform is python.

\section{REFERENCE:}

[1] A. Shahab, F. Shafait, and A. Dengel, "ICDAR 2011 robust reading competition: ICDAR Robust Reading Competition Challenge 2: Reading text in scene images," in Proc. Int. Conf. Document Anal. Recognition,2011, pp. 1491-1496.

[2] Advance Data Reports from the National Health Interview Survey (2008).[Online]. Available: http://www.cdc.gov/nchs/nhis/nhis_ad.htm.

[3] B. Epshtein, E. Ofek, and Y. Wexler, "Detecting text in natural scenes with stroke width transform," in Proc. Comput. Vision Pattern Recognition, 2010, pp. 2963-2970.

[4] C. Yi and Y. Tian, "Assistive text reading from complex background for blind persons," in Proc. Int. Workshop Camera-Based Document Anal.Recognit., 2011, vol. LNCS-7139, pp. 15-28.

[5] C. Yi and Y. Tian, "Text string detection from natural scenes by structure based partition and grouping," IEEE Trans. Image Process., vol. 20, no. 9, pp. 2594-2605, Sep. 2011.

[6] International Workshop on Camera-Based Document Analysis and Recognition (CBDAR 2005, 2007, 2009, 2011). [Online]. Available: http://www.m.cs.osakafuu.ac.jp/cbdar2011/

[7] J. Zhang and R. Kasturi, "Extraction of text objects in video documents: recent progress," in Proc. IAPR Workshop Document Anal. Syst., 2008, pp. 5-17.57

[8] K. Kim, K. Jung, and J. Kim, "Texture-based approach for text detection in images Using support vector machines and continuously adaptive mean shift algorithm," IEEE Trans. Pattern Anal. Mach. Intel., vol. 25, no. 12, pp. 1631-1639, Dec. 2003.

[9] L. Ma, C. Wang, and B. Xiao, "Text detection in natural images based on multi-scale edge detection and classification,” in Proc. Int. Congr.Image Signal Process., 2010, vol.4, pp. 1961-1965.

[10]N.Nikolaou and N. Papamarkos, "Color reduction for complex document images," Int. J. Imaging Syst. Technol., vol. 19, pp. 14-26, 2009. 